\title{
Use of Shock Index and Lactate to Predict Mortality in Acute Heart Failure Patients in Emergency Department
}

\author{
Hasan Basri Cetinkaya and Harun Gunes \\ Department of Emergency Medicine, Balikesir University School of Medicine, Balikesir, Turkey
}

\begin{abstract}
Objective: To compare the usefulness of shock index (SI) and lactate for prediction of 24-hour and 28-day mortality in acute heart failure (AHF) patients.

Study Design: A descriptive study.

Place and Duration of the Study: Balikesir University Hospital, Balikesir, Turkey; from February 2019 to August 2020.

Methodology: One hundred and twelve AHF patients presenting to ED were recruited into the study. Usefulness of lactate and SI in predicting mortality at 24-hour and 28-day, was evaluated.

Results: The area under the curve (AUC) was found to be 0.825 for lactate and 0.818 for SI in predicting 24 -hour mortality. There was significant difference between the diagnostic performances of 2 markers in predicting 24-hour mortality. AUC was found to be 0.775 for lactate and 0.722 for $\mathrm{SI}$ in predicting 28-day mortality. No significant difference was found between the diagnostic performances of the two markers in predicting 28-day mortality. The 24 -hour non-survivor rates were found to be $86.67 \%$ in patients with lactate levels $>2.57 ; 76.47 \%$ in patients with $\mathrm{SI}>0.94$, and $93.33 \%$ in patients with lactate levels $>2.57$ or $\mathrm{SI}>0.94$. The 28 -day non-survivor rates were found to be $64.71 \%$ in patients with lactate levels $>2.57 ; 70.59 \%$ in patients with $\mathrm{SI}>0.82$, and $82.35 \%$ in patients with lactate levels $>2.57$ or $\mathrm{SI}>0.82$.

Conclusion: Lactate level and SI of AHF patients calculated in ED triage may be used to predict mortality, and simultaneous use of both parameters may be more helpful.
\end{abstract}

Key Words: Acute heart failure, Emergency department, Lactate, Mortality, Shock index.

How to cite this article: Cetinkaya HB, Gunes H. Use of Shock Index and Lactate to Predict Mortality in Acute Heart Failure Patients in Emergency Department. J Coll Physicians Surg Pak 2021; 31(03):262-266.

\section{INTRODUCTION}

Signs and symptoms of acute heart failure (AHF) may occur abruptly or develop gradually. The patient needs emergent intervention in both scenarios, because the condition of the patient may deteriorate to pulmonary edema and cardiogenic shock. ${ }^{1} \mathrm{AHF}$ is the leading cause of hospitalisations in the elderly in the western world. It still has an unacceptable morbidity and mortality rate despite recent improvements in the medical treatment and mechanical ventilation options. In-hospital mortality rate is $4-7 \%$; re-hospitalisation rate is $25-30 \%$, and mortality rate is $7-11 \%$ within 3 months of discharge from the hospital. Hence, AHF causes a huge economic burden by becoming a public health problem. ${ }^{2}$ Early detection of low risk patients may prevent unnecessary hospitalisations and decrease economic costs. ${ }^{3,4}$

Correspondence to: Dr. Hasan Basri Cetinkaya, Department of Emergency Medicine, Balikesir University School of Medicine, Balikesir, Turkey

E-mail: hbcetinkaya@gmail.com

Received: October 05, 2020; Revised: February 15, 2021;

Accepted: February 19, 2021

DOI: https://doi.org/10.29271/jcpsp.2021.03.262
Besides, determination of high risk patients is critical for timely initiation of appropriate treatment and reduction of the mortality rate. ${ }^{5}$ Thus, having a simple tool, which can discriminate low-risk patients from those with high-risk, would be helpful.

Elevated blood lactate level shows tissue hypo-perfusion, which is a strong prognostic factor. Lactate level is commonly used in therapeutic algorithms in intensive care medicine.$^{6-8}$ Peripheral hypo-perfusion, as a result of lower cardiac output, higher central venous pressure and vasoconstriction due to increased sympathetic activity, leads to deterioration of production-clearance balance of lactate in AHF. ${ }^{9}$

Shock index (SI), which can be calculated at the bedside, had initially been used to evaluate patients with several clinical conditions, causing cardiovascular compromise such as hemorrhage and pulmonary embolism. ${ }^{10,11}$ Although SI may be affected by the patient's general clinical condition and the medications he uses, it gives the physician the opportunity to evaluate the patient's risk status in the Emergency Department. ${ }^{12-14}$ SI has been used for risk stratification in various intensive care unit settings, and it was shown that elevated levels are independent predictors of mortality, micro-vascularinjury, and myocardial damage..$^{10,11,13}$ 
Although lactate and SI had been used in different clinical scenarios, as far as it is seen, they were not tested for their mortality-predictive potential in AHF patients in Emergency Department. Thus, it was aimed, in the present study, to evaluate and compare predictive power of SI and lactate measured in the Emergency Department triage when determining 24hour and 28-day mortality risk in AHF patients. Besides, it was aimed to evaluate whether simultaneous use of these parameters has a higher predictive potential.

\section{METHODOLOGY}

This descriptive study was performed in Balikesir University Hospital, Balikesir, Turkey. First, the patients who were diagnosed with AHF in the Emergency Department between February 1, 2019 and August 1, 2020 were determined by carefully checking the medical records of all Emergency Department patients retrospectively. Those patients were recruited into the study, if they did not have any exclusion criterion. The first exclusion criterion was being below 18 years of age. Patients presenting with any type of trauma, and those for whom sufficient information regarding the clinical status 24 hours and 28 days after the admission could not be obtained, were excluded, too. AHF diagnosis was established by the emergency physician and confirmed by the consulting cardiologist. Those patients in whom cardiologist did not confirm the diagnosis of AHF and the patients who had a history of congestive heart failure but presented to the Emergency Department for complaint not related to heartfailure, were excluded.

The patients' blood pressure (BP), pulse rate (PR), and lactate levels were taken from their medical records. Lactate levels of five patients could not be found in the records. It was determined whether the patient survived 24 hours and 28 days after admission by investigating his or her clinical records in the hospital files; or information on E-Nabizsystem of the Ministry of Health, when the hospital records did not have sufficient information. E-Nabiz system is a country-wide, web-based system which includes information about the patient's diseases, prescribed medications, results of laboratory tests and imaging studies; and death information, if the patient died. Survival status of a patient was evaluated using the information in the hospital records and E-Nabiz system. If the hospital records include any information about the death of the patient during the first 24 hours or 28 days after admission to the Emergency Department, the patient was included in the dead group, otherwise information in the E-Nabiz system was also checked; and the patient was included in the dead group, if he died in another hospital or in his home or somewhere else. If neither the hospital records nor E-Nabiz system include any information about the death of the patient, the patient was included in the alive group. The patients, whose survival information could not be reached, were excluded from the study. SI was calculated as PR divided by systolic blood pressure (SBP). Blood pressure measurements were performed using GE Carescape V100 Dinamap Vital Signs Monitor (GE Medical Systems Information Technologies Inc., Milwaukee, Wisconsin, USA) in the Emergency Department triage room. Epoc Blood Analysis Epoc Reader (Epocal Inc., Ottawa, Canada) device was used for arterial blood gas analyses. The patients' age, gender, and 24-hour and 28-day survival status (dead or alive) were recorded. This retrospective chart review study involving human participants was in accordance with the ethical standards of the Institutional and National Research Committee and 1964 Helsinki Declaration and its later amendments or comparable ethical standards. The Local Ethics Committee approved this study (Decision Date: August 19, 2020, Decision Number: 2020/141).

Shapiro-Wilk test was used to test the normality of variables. Continuous variables were presented as mean \pm standard deviation for normally distributed variables; otherwise, median (25th percentile-75th percentile) values were given. Independent samples t-test was performed for comparison of two independent groups, if the data was normally distributed; otherwise, Mann-Whitney's U-test was used for comparison of two groups. Categorical variables were expressed by numbers and percentages. Comparisons between the groups were performed with Pearson Chi-square test for categorical variables. Receiver operating characteristics (ROC) curve analysis was performed to evaluate and compare the performances of diagnostic markers. Significance level was taken as $\alpha=0.05$. Statistical analyses were performed with IBM SPSS Statistics version 22.0 (IBM Corp., Armonk, NY, USA) and MedCalc version 12.3.0.0.

\section{RESULTS}

Fifty-four patients (48.21\%) were males and 58 patients $(51.79 \%)$ were females. Mean age of the patients was found $74.88 \pm 9.45$ years. Median values of lactate and SI were 1.80 $\mathrm{mmol} / \mathrm{L}$ (25th percentile-75th percentile: 1.08-3.20) and 0.78 (25th percentile-75th percentile: 0.57-0.97), respectively. Twenty-four-hour and 28-day mortality rates were $15.18 \%(n=17)$ and $34.82 \%(n=39)$, respectively.

Gender distribution and mean age of the patients were found not differential significantly between survivors and non-survivors of the first 24-hour-period ( $p=1.000$ and $p=0.869$, respectively). The same was also true for 28-day mortality $(p=0.605$ and $p=0.245$, respectively). Lactate and SI values were significantly higher in non-survivors than in survivors for both 24 hours and 28 days (Tablel).

ROC curve analyses were performed to evaluate the diagnostic performances of lactate and SI in predicting 24-hour and 28-day mortality. Optimal cut-off values were obtained according to Youden J index. It was $2.57 \mathrm{mmol} / \mathrm{L}$ for lactate and 0.94 for SI. Corresponding sensitivity and specificity values were calculated. Sensitivities of lactate and SI were $86.67 \%$ and $80.00 \%$ for 24-hour outcome, respectively while they were $64.71 \%$ and $70.59 \%$ for 28 -day outcome, respectively. Specificities of them were $75.00 \%$ and $84.78 \%$ for 24 -hour outcome, respectively; they were $80.82 \%$ and $75.34 \%$ for 28 -day outcome, respectively. The area under the curve (AUC) was found as $0.825(p<0.001)$ for lactate and $0.818(p<0.001)$ for $\mathrm{SI}$ in predicting 24-hour mortality. 
Table 1: Comparison of lactate and shock index values in terms of 24-hour and 28-day mortality.

\begin{tabular}{|l|l|l|l|l|}
\hline Period & Variable & Survivor & Non-survivor & p-value \\
\hline \multirow{2}{*}{$24-$ Hours } & Lactate $(\mathrm{mmol} / \mathrm{L})^{*}$ & $1.59(1.00-2.70)$ & $6.50(2.93-11.34)$ & $<0.001$ \\
\cline { 2 - 5 } & Shock index* & $0.75(0.56-0.90)$ & $1.11(0.91-1.41)$ & 0.001 \\
\hline \multirow{2}{*}{$28-$-Days } & Lactate $\left(\mathrm{mmol}^{*} / \mathrm{L}\right)^{*}$ & $1.46(0.90-2.47)$ & $3.41(1.75-7.23)$ & $<0.001$ \\
\cline { 2 - 5 } & Shock index* & $0.71 \pm 0.21$ & $1.04 \pm 0.42$ & $<0.001$ \\
\hline \multicolumn{2}{|l|}{ Data given as ${ }^{*}$ mean \pm standard deviation or ${ }^{*}$ median (25th percentile-75th percentile). } & \\
\hline
\end{tabular}

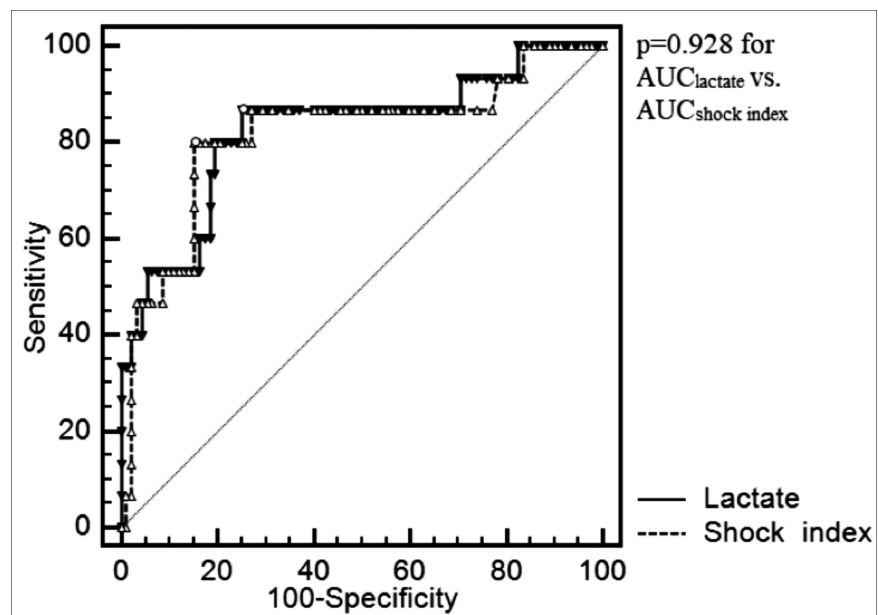

Figure 1: ROC curves of lactate and shock index for 24-hour mortality.

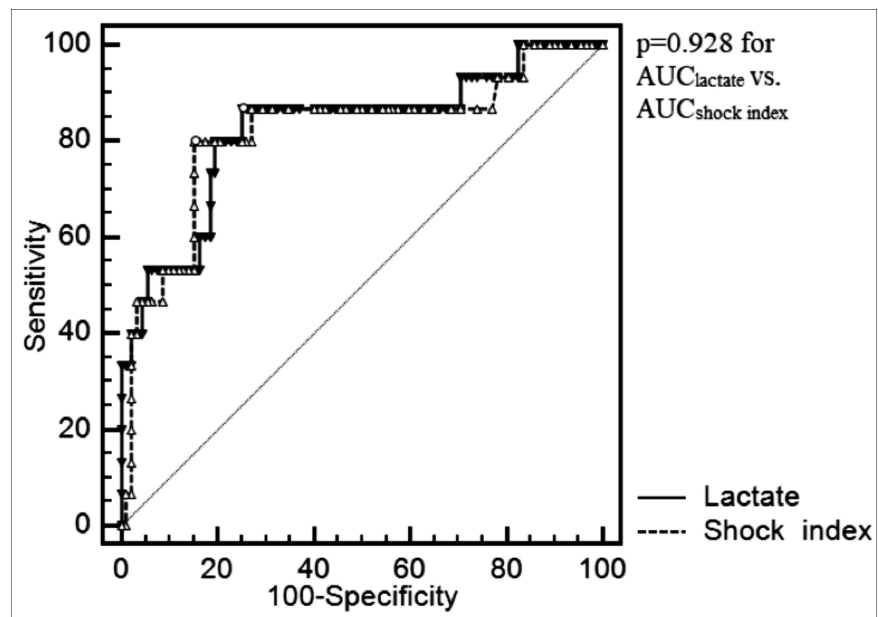

Figure 2: ROC curves of lactate and shock index for 28-day mortality.

Table II: Distribution of categorised lactate and shock index values, according to 24-hour and 28-day outcomes.

\begin{tabular}{|c|c|c|c|}
\hline \multirow{2}{*}{\multicolumn{2}{|c|}{ Variable }} & \multicolumn{2}{|c|}{ 24-hour outcome [n (\%)] } \\
\hline & & Dead & Alive \\
\hline \multirow{2}{*}{ Lactate $(\mathrm{mmol} / \mathrm{L})$} & $>2.57$ & $13(86.67)$ & $24(26.09)$ \\
\hline & $<=2.57$ & $2(13.33)$ & $68(73.91)$ \\
\hline \multirow{2}{*}{ Shock index } & $>0.94$ & $13(76.47)$ & $18(18.95)$ \\
\hline & $<=0.94$ & $4(23.53)$ & 77 (81.05) \\
\hline \multirow{2}{*}{$\begin{array}{l}\text { Lactate and shock index } \\
\text { together }\end{array}$} & $>2.57$ or $>0.94$ & $14(93.33)$ & $33(35.87)$ \\
\hline & Others & $1(6.67)$ & $59(64.13)$ \\
\hline & & \multicolumn{2}{|c|}{ 28-day outcome [n (\%)] } \\
\hline & & Dead & Alive \\
\hline \multirow{2}{*}{ Lactate $(\mathrm{mmol} / \mathrm{L})$} & $>2.57$ & $22(64.71)$ & $15(20.55)$ \\
\hline & $<=2.57$ & $12(35.29)$ & $58(79.45)$ \\
\hline \multirow{2}{*}{ Shock index } & $>0.82$ & $28(71.79)$ & $20(27.40)$ \\
\hline & $<=0.82$ & $11(28.11)$ & $53(72.60)$ \\
\hline \multirow{2}{*}{$\begin{array}{l}\text { Lactate and shock index } \\
\text { together }\end{array}$} & $>2.57$ or $>0.82$ & $28(82.35)$ & $33(45.21)$ \\
\hline & Others & $6(17.65)$ & $40(54.80)$ \\
\hline
\end{tabular}

There was not a significant difference between the diagnostic performances of the two markers in predicting 24hour mortality $(p=0.928)$. AUC was found $0.775(p<0.001)$ for lactate and $0.722(p<0.001)$ for $S I$ in predicting 28-day mortality. The difference between the diagnostic performances of two markers in predicting 28-day mortality was not significant ( $p=0.404$, Figures 1 and 2$)$.

Lactate and SI were also categorised according to their cutoff values calculated from the ROC curve analysis. The 24hour non-survivor rates were found to be $86.67 \%(n=13)$ in patients with lactate levels, higher than $2.57 ; 76.47 \%$ $(n=13)$ in patients with SI levels higher than 0.94 , and $93.33 \%(n=14)$ in patients with lactate levels higher than 2.57 or SI levels higher than 0.94 . The 28 -day non-survivor rates were found to be $64.71 \%(n=22)$ in patients with lactate levels higher than $2.57 ; 71.79 \%(n=28)$ in patients with SI levels higher than 0.82 , and $82.35 \%(n=28)$ in patients with lactate levels higher than 2.57 or SI levels higher than 0.82 (Table II).

\section{DISCUSSION}

AHF still has a high mortality rate despite the improvements in the diagnosis and management. Simple parameters, which can discriminate the patients with critical clinical situation from those without such a condition in a crowded Emergency Department are needed. When those with a critical condition are determined early, it means they can be given appropriate treatment earlier. ${ }^{15}$

There are several studies indicating that lactate levels can be used to predict mortality in septic shock, post-cardiac arrest period and multiple trauma victims. ${ }^{6-8}$ Elevation of lactate levels, as a result of the deterioration of the balance between the production and elimination of lactate, may occur in AHF patients, too. ${ }^{9}$ Different cut-off values were determined in different studies performed to predict the performance of lactate in predicting mortality in heart failure patients. Cut-off values were determined by authors themselves in some studies and calculated using statistical tests in other studies. The studies on this topic have some other methodological differences, too. For example, some studies performed ROC analysis and calculated AUC but others did not. Uyar et al. evaluated 6-month mortality rates in AHF patients with a cutoff value of $2 \mathrm{mmol} / \mathrm{L}$ for lactate, and they did not find a significant difference between the groups. ${ }^{16}$ Gjestal et al. found that a $2.5 \mathrm{mmol} / \mathrm{L}$ cut-off value of lactate provides additional prognostic information in mild to moderate heart failure patients. They saw that 30 -day mortality rate was $28 \%$ in the group with lactate above $2.5 \mathrm{mmol} / \mathrm{L}^{17}$ In the present study, 
28-day mortality rate was found $59.45 \%$ when the lactate level was above cut-off value of $2.57 \mathrm{mmol} / \mathrm{L}$. Although there are slight differences between the cut-off values of lactate ( $2.5 \mathrm{mmol} / \mathrm{L}$ vs. $2.57 \mathrm{mmol} / \mathrm{L}$ ) and periods evaluated (30 days vs 28 days) in these two studies, the authors think the major reason of the difference between the mortality rates was patient characteristics. The study by Gjestal et al. evaluated mild to moderate heart failure patients, but all AHF patients (including those with cardiogenic shock) were recruited into the present study. Besides, mean age of the patients was higher in the present study compared to that of the study by Gjestal et al. (75 years vs. 67 years). Kawasake et al. saw that elevated arterial lactate levels in heart failure patients are associated with a worsened mortality rate when there was no time limit. They found the best cut-off value of lactate as $3.2 \mathrm{mmol} / \mathrm{L}$ with a sensitivity and specificity of $60.2 \%$ and $74.6 \%$, respectively. And they suggested that an elevated lactate level may help predict the risk of early death. ${ }^{18}$ Desmolin et al. evaluated 30-day mortality risk and found mean lactate levels $1.14 \mathrm{mmol} / \mathrm{L}$ and $1.78 \mathrm{mmol} / \mathrm{L}$ in survivors and non-survivors, respectively. ROC analysis had shown an AUC of 0.73 in that study. ${ }^{19}$ In the present study, which evaluated 28-day mortality rate, mean lactate levels were found $1.46 \mathrm{mmol} / \mathrm{L}$ and $3.41 \mathrm{mmol} / \mathrm{L}$ in survivors and non-survivors, respectively. And ROC analysis showed a similar AUC (0.77) to that of the study by Desmolin et al.

The mortality-predictive performance of SI was evaluated in the current study, too. The first study investigating the possible mortality-predictive potential of SI in AHF patients has been performed by Pourafkari et al. They did not found a significant relationship between SI and neither in-hospital nor long-term mortality. ${ }^{20}$ Whereas, El Menyar et al. found, in a recent cohort study, that a cut-off value of 0,9 for SI may be useful in predicting in-hospital and 3-month mortality. ${ }^{21}$ However, sensitivity and specificity in predicting in-hospital mortality were found to be $49 \%$ and $79 \%$, respectively. The sensitivity was too low to recommend the use of cut-off value of 0.9 for prediction of mortality. In the present study, SI was much reliable in predicting 24-hour and 28-day mortality when the cut-off value was 0.94 and 0.82 , respectively. A cutoff value of 0.94 resulted in $80 \%$ sensitivity and $84.7 \%$ specificity when predicting 24-hour mortality risk, and a cutoff value of 0.82 has $70.5 \%$ sensitivity and $75.3 \%$ specificity when predicting 28-day mortality risk.

Although both lactate and SI were seen to individually predict mortality in AHF patients, it was also evaluated, in the present study, whether simultaneous use of these two parameters provide more reliable predictive power. As far as the authors could search, there was no study evaluating the mortality-predictive potential of simultaneous use of lactate and $\mathrm{SI}$ in the English literature. Lactate and SI could predict $86.6 \%$ and $76 \%$ of deaths occurring during the first 24 hours on themselves, respectively. When predictive potential of simultaneous use of both parameters was evaluated, it was seen that they can predict $93.3 \%$ of deaths. For the 28 -day mortality, lactate and SI could individually predict $64.7 \%$ and $70.5 \%$ of deaths, respectively. When their combined use was evaluated, they could predict $82.3 \%$ of deaths. As mentioned previously, the English literature lacks any previous studies to compare our results with. It is suggested that using lactate and $\mathrm{SI}$ together may be more helpful when predicting both 24-hour and 28-day mortality risk in AHF patients.

The current study has some limitations. First, it is a retrospective study so the patients' clinical course could not be followed and any additional intervening factors in this course could not be detected. The second one is relatively small number of subjects, but files of all possible AHF patients presenting to the Emergency Department since the opening of the Emergency Department were investigated (the Emergency Department opened in February 2019, and the number of Emergency Department admissions is fairly low since the hospital is nearly 20 kilometers away from the city), and all patients meeting inclusion criteria and not having any exclusion criterion were recruited into the study.

\section{CONCLUSION}

Lactate and SI calculated during Emergency Department triage may be helpful individually in predicting 24-hour and 28-day mortality risk in AHF patients. None of these parameters is superior to the other, and physicians may better consider using these two parameters together to obtain a more reliable predictive power.

\section{ACKOWLEDGEMENT:}

The authors would like to thank Associate Professor Deniz Sigirli of Bursa Uludag University School of Medicine Biostatistics Department for her invaluable help in doing statistical analyses.

\section{ETHICAL APPROVAL:}

This retrospective chart review study involving human participants was in accordance with the ethical standards of the Institutional and National Research Committee; and with 1964 Helsinki Declaration and its later amendments or comparable ethical standards. Balikesir University Clinical Studies Ethics Committee approved this study (Decision Date: August 19, 2020, Decision No. 2020/141).

\section{PATIENTS' CONSENT:}

This study was a retrospective study and the information for the study was gathered by scanning the hospital records after Ethics Committee approval.

\section{CONFLICT OF INTEREST:}

The authors declared no conflict of interest.

\section{AUTHORS' CONTRIBUTION:}

HBC, HG: Contributed to the study conception and design, material preparation and data collection. Analysis of the data was performed with the help of Associate Professor Deniz Sigirli of Bursa Uludag University School of Medicine 
Biostatistics Department, Bursa, Turkey. The first draft of the manuscript was written by Hasan Basri Cetinkaya, and Harun Gunes commented on previous versions of the manuscript. Both authors read and approved the final manuscript.

\section{REFERENCES}

1. Ponikowski P, Voors AA, Anker SD, Bueno H, Cleland JGF, Coats AJS, et al. 2016 ESC guidelines for the diagnosis and treatment of acute and chronic heart failure: The task force for the diagnosis and treatment of acute and chronic heart failure of the European Society of Cardiology (ESC), developed with the special contribution of the Heart Failure Association (HFA) of the ESC. Eur Heart J 2016; 37(27): 2129-200. http://doi.org/10.1093/eurheartj/ehw128.

2. Farmakis D, Parissis J, Filippatos G. Acute heart failure: Epidemiology, classification and pathophysiology. In: Tubaro M, Vranckx P, Price S, Vrints C, Eds. The ESC textbook of intensive and acute cardiovascular care, 2nd ed. Oxford; Oxford University Press; 2015: p. 459-69.

3. Collins SP, Lindsell CJ, Jenkins CA, Harrell FE, Fermann GJ, Miller KF, et al. Risk stratification in acute heart failure: Rationale and design of the stratify and decide studies. Am Heart J. 2012; 164(6):825-34. http://doi.org/10.1016/ j.ahj.2012.07.033.

4. Collins SP, Pang PS, Fonarow GC, Yancy CW, Bonow RO, Gheorghiade $M$. Is hospital admission for heart failure really necessary? The role of the ED and observation unit in preventing hospitalization and rehospitalization. J Am Coll Cardiol 2013; 61 (2):121-6. http://doi.org/10.1016/j.jacc.2012.08.1022.

5. Lee DS, Austin PC, Rouleau JL, Liu PP, Naimark D, Tu JV. Predicting mortality among patients hospitalized for heart failure: derivation and validation of a clinical model. JAMA 2003; 290(19):2581-7. http://doi.org/10.1001/jama.290. 19.2581.

6. Nichol A, Bailey M, Egi M, Pettila V, French C, Stachowski E, et al. Dynamic lactate indices as predictors of outcome in critically ill patients. Crit Care 2011; 15:242-52. http://doi.org/10.1186/cc10497.

7. Meregalli A, Oliveira RP, Friedman G. Occult hypoperfusion is associated with increased mortality in hemodynamically stable, high-risk, surgical patients. Crit Care 2004; 8(2):60-5. http://ccforum.com/content/8/2/R60.

8. Kliegel A, Losert H, Sterz F, Holzer M, Zeiner A, Havel C, et al. Serial lactate determinations for prediction of outcome after cardiac arrest. Medicine 2004; 83:274-9. http://doi.org/10.1097/01.md.0000141098.46118.4c.

9. Orn S, van Hall G. Does a normal peripheral lactate value always indicate an aerobic tissue metabolism? Eur J Heart Fail 2017; 19:1034-5. http://doi.org/10.1002/ejhf.863.

10. El-Menyar A, Goyal P, Tilley E, Latifi R. The clinical utility of shock index to predict the need for blood transfusion and outcomes in trauma. J Surg Res 2018; 227:52-9. http://doi.org/10.1016/j.jss.2018.02.013.

11. Ozsu S, Erbay M, Durmus ZG, Ozlu T. Classification of high-risk with cardiac troponin and shock index in normotensive patients with pulmonary embolism. J Thromb Thrombolysis 2017; 43(2):179-83. http://doi.org/10.1007/s11239-0161443-3.

12. Yu T, Tian C, Song J, He D, Sun Z, Sun Z. Derivation and validation of shock index as a parameter for predicting longterm prognosis in patients with acute coronary syndrome. Sci Rep 2017; 7(1):11929. http://doi.org/ 10.1038/s41598-017-12180-2.

13. Reinstadler SJ, Fuernau G, Eitel C, de Waha S, Desch S, Metzler $\mathrm{B}$, et al. Shock index as a predictor of myocardial damage and clinical outcome in ST-elevation myocardial infarction. Circ J 2016; 80(4):924-30. http://doi.org/10. 1253/circj.CJ-15-1135.

14. Berger T, Green J, Horeczko T, Hagar Y, Garg N, Suarez A, et al. Shock index and early recognition of sepsis in the emergency department: pilot study. West J Emerg Med 2013; 14(2):168-74. http://doi.org/10.5811/westjem.2012.8.11546.

15. Mehmood A, He S, Zafar W, Baig N, Sumalani F, Razzak J. How vital are the vital signs? a multi-center observational study from emergency departments of Pakistan. BMC Emerg Med 2015; 15(Suppl 2):S10. http://doi.org/10. 1186/1471-227X-15-S2-S10.

16. Uyar H, Yesil E, Karadeniz M, Orscelik O, Ozkan B, Ozcan T, et al. The effect of high lactate level on mortality in acute heart failure patients with reduced ejection fraction without cardiogenic Shock. Cardiovasc Toxicol 2020; 20:361-9. http://doi.org/10.1007/s12012-020-09563-9.

17. Gjesdal G, Braun OÖ, Smith JG, Scherstén F, Tydén P. Blood lactate is a predictor of short-term mortality in patients with myocardial infarction complicated by heart failure but without cardiogenic shock. BMC Cardiovasc Disord 2018; 18(1):8. http://doi.org/10.1186/s12872-018-0744-1.

18. Kawase T, Toyofuku M, Higashihara T, Masaoka Y, Shiode N, Hayashi $Y$, et al. Validation of lactate level as a predictor of early mortality in acute decompensated heart failure patients who entered intensive care unit. J Cardiol 2015; 65(2):164-70. http://doi.org/10.1016/j.jjcc.2014.05.006.

19. Desmoulin F, Galinier M, Trouillet C, Berry M, Delmas C, Turkieh $A$, et al. Metabonomics analysis of plasma reveals the lactate to cholesterol ratio as an independent prognostic factor of short-term mortality in acute heart failure. PLoS One 2013; 8(4):e60737. http://doi.org/ 10.1371/journal. pone.0060737.

20. Pourafkari L, Wang CK, Schwartz M, Nader ND. Does shock index provide prognostic information in acute heart failure? Int J Cardiol 2016; 215:140-2. http://doi.org/10.1016/j.ijcard.2016.04.083.

21. El-Menyar A, Sulaiman K, Almahmeed W, Al-Motarreb A, Asaad N, AlHabib KF, et al. Shock index in patients presenting with acute heart failure: A multicenter multinational observational study. Angiology 2019; 70(10):938-46. http://doi.org/10.1177/0003319719857560. 